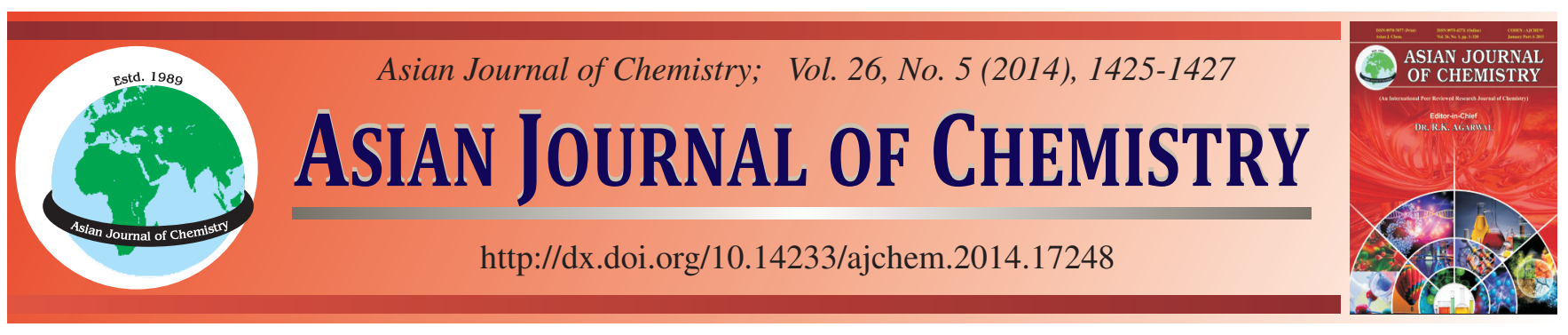

\title{
Preparation and Performance of Urea-Formaldehyde Resin Modified with Phenolized Fulvic Acid and Isocyanate $\nmid$
}

\author{
Xianglin Zhang ", Jun Xu, Ronguia Dai and Houwei Chen
}

School of Material and Chemical Engineering, Anhui Jianzhu University, Hefei 230022, P.R. China

*Corresponding author: Tel: +86 13705513262; E-mail: zxlaua@163.com

Urea-formaldehyde (UF) resins were prepared by using phenolized fulvic acid (PFA) and 4,4'-diphenylmethane diisocyanate (MDI) as modifiers through orthogonal experiment. The influence of the PFA/UF ratio (mass ratio) on free formaldehyde and that of the MDI/UF ratio on bonding strength were investigated. The results showed that the content of free formaldehyde decreased with an increasing PFA/ UF ratio and the bonding strength increased with an increasing MDI/UF ratio. When the mass ratio of PFA:MDI:UF was 0.006:0.035:1, the free formaldehyde content was dropped to $0.0924 \%$, the bonding strength could be achieved at $1.08 \mathrm{MPa}$ and the thermal stability was also improved accordingly.

Keywords: Urea-formaldehyde resins, Free formaldehyde, Phenolized fulvic acid, Isocyanate, Bonding strength.

\section{INTRODUCTION}

Urea-formaldehyde (UF) resins are based on the reaction of urea and formaldehyde. Due to low cost, excellent adhesion to wood and lack of colour in the finished product, ureaformaldehyde resins are become the most widely used adhesives for wood-based products. However, formaldehyde is easily released since the wood-based products will be used in a long term ${ }^{1,2}$, which can pollute the indoor environment seriously $^{3}$ and endanger human health ${ }^{4,5}$. The emission of formaldehyde mainly depends on the content of free formaldehyde of resins ${ }^{6}$. So urea-formaldehyde resins with low content of free formaldehyde are urgently needed.

Fulvic acid (FA) is one kind of natural organic material, which contains several kinds of aliphatic and aromatic active functional groups such as hydroxyl, amino, methylol and phenols. This paper used phenol to prepare phenolized fulvic acid (PFA) for the purpose of improving the reactivity with formaldehyde.

Que et al. ${ }^{7}$, Pizzi et $a .^{8}{ }^{8}$ and Myers ${ }^{9}$ reported that the emission of formaldehyde from wood products decreased as the mole ratio (formaldehyde/urea) falls, but unfortunately, the mechanical properties were influenced negatively at the same time. All urea-formaldehyde resins used for this paper were prepared in the laboratory, following traditional alkaline-acid-alkaline three-step reaction. The purpose of this study was to prepare ureaformaldehyde resins with low content of free formaldehyde and good bonding strength.

\section{EXPERIMENTAL}

Phenolized fulvic acid preparation: The lignite and excess of distilled water were placed in the reactor, the $\mathrm{pH}$ was corrected by addition of $20 \%$ (mass ratio) sodium hydroxide solution to 10 after stirring for $15 \mathrm{~min}$. The temperature raised to $80{ }^{\circ} \mathrm{C}$ and maintained for $3 \mathrm{~h}$. Then the mixture was centrifuged at $4000 \mathrm{rpm}$ for $20 \mathrm{~min}$ to get the solution. Using $20 \%$ hydrochloric acid solution to acidify the solution to $\mathrm{pH}$ 1-2 and then centrifuged at $5000 \mathrm{rpm}$ for $20 \mathrm{~min}$ to collect the supernatant. The fulvic acid was obtained after the supernatant was dried at $65^{\circ} \mathrm{C}$.

$25 \mathrm{~g}$ Fulvic acid, $10 \mathrm{~g}$ phenol and excess of deionized water were placed in another reactor. The mixture was heated to $65^{\circ} \mathrm{C}$ in $0.5 \mathrm{~h}$ where the $\mathrm{pH}$ was adjusted to 10 with $20 \%$ $\mathrm{NaOH}$ solution. The temperature raised to $85^{\circ} \mathrm{C}$ in $15 \mathrm{~min}$ and maintained at $85^{\circ} \mathrm{C}$ for $3 \mathrm{~h}$, then the mixture was dried at $65^{\circ} \mathrm{C}$ to get the phenolized fulvic acid.

Orthogonal experiment of urea-formaldehyde resins: $70 \mathrm{~g}$ by mass of $37 \%$ formaldehyde solution was placed in the reactor, the temperature raised to $45^{\circ} \mathrm{C}$ and maintained for $10 \mathrm{~min}$. A certain amount $\left(\mathrm{n}_{\mathrm{F}} / \mathrm{n}_{\mathrm{U}}\right.$, factor $\mathrm{A}$ of the orthogonal

†Presented at The 7th International Conference on Multi-functional Materials and Applications, held on 22-24 November 2013, Anhui University of Science \& Technology, Huainan, Anhui Province, P.R. China 
experiment) of urea was divided into three equal parts, then the first part of urea was added to the reactor after $\mathrm{pH}$ was adjusted to 8 with $20 \% \mathrm{NaOH}$ solution. The mixture was heated to $80^{\circ} \mathrm{C}$ in $0.5 \mathrm{~h}$ and maintained at $80^{\circ} \mathrm{C}$ for a certain time $\left(\mathrm{t}_{1}\right.$, factor $\mathrm{B}$ ). Adjust $\mathrm{pH}$ to a given value ( $\mathrm{pH}$, factor $\mathrm{C}$ ) with solution prepared by $10 \%$ ammonium chloride and $1 \mathrm{~mol} / \mathrm{L}$ hydrochloric acid at the volume ratio of 1:5, then added the second part of urea and kept the temperature to a given value ( $\mathrm{T}$, factor D) for a certain time $\left(\mathrm{t}_{2}\right.$, factor $\left.\mathrm{E}\right)$. The $\mathrm{pH}$ was adjusted to 8 with solution prepared by $10 \%$ ammonia and $1 \mathrm{~mol} / \mathrm{L}$ sodium hydroxide at the volume ratio of $1: 5$, then added the last part of urea to keep reacting for $0.5 \mathrm{~h}$ at $80{ }^{\circ} \mathrm{C}$. The UF resins was obtained after the mixture was cooled to room temperature.

PFA-MDI modified UF resins preparation: Factor A, B, C, D and E were determined after orthogonal experiment. A certain value of PFA was added after the first part of urea and $0.5 \%$ (accounting for the mass of urea and formaldehyde) MDI was added after the second part of urea, then PFA-MDI modified UF (PMUF) resins were obtained based on the optimal conditions.

Urea-formaldehyde resins characterization: Free formaldehyde content of the UF resins was tested according to the ISO 11402-2004. Bonging strength was tested according to GB/T 14074-2006. Laminates with a size of 360 (L) $\times 360$ (W) $\times 10(\mathrm{~T}) \mathrm{mm}$ were prepared using each of 5 different PMUF resins adhesives. A certain mass of PMUF resins, 1 \% (accounting for the mass of urea and formaldehyde) ammonium chloride, $5 \%$ flour and a given mass of MDI was added in a react to prepare PMUF adhesives which were supplied to the sheets in a coater, $320 \mathrm{~g} / \mathrm{sm}$. Laminates were produced in a pressing machine and hot-press at $1.1 \mathrm{MPa}$ for $5 \mathrm{~min}$ and temperatures of $120{ }^{\circ} \mathrm{C}$.

\section{RESULTS AND DISCUSSION}

Free formaldehyde content results and discussion: As listed in Table-1, factors produced a huge impact on the content of free formaldehyde, especially the factor A. The optimal conditions of the UF resins are as follows: $\mathrm{n}_{\mathrm{F}} / \mathrm{n}_{\mathrm{U}}=1.0, \mathrm{t}_{1}=80$ min, $\mathrm{pH}=4.5, \mathrm{~T}=85^{\circ} \mathrm{C}, \mathrm{t}_{2}=80 \mathrm{~min}$. However, the optimal conditions were not listed in the table, additional experiment must to be conducted and the content of free formaldehyde was $0.135 \%$ lower that other groups.

Fig. 1 clearly showed that PFA can effectively reduce the free formaldehyde content of UF resins. But when the content of PFA/UF is over $0.6 \%$, free formaldehyde content is essentially unchanged. Taking into account of the negatively impact with the colour of UF resins, $0.6 \%$ is a more reasonable choice and the content of the free formaldehyde is $0.0924 \%$ at this point.

Bonding strength: Fig. 2 showed the bonding strength of the laminates at different MDI/UF mass ratios. As the increase of mass ratio, the bonding strength increases significantly. It is mainly because MDI contains active isocyanate groups, which could carry out crosslinked action with active hydrogen groups such as hydroxyl and amino group, covalent bond will be formed between resins and wood materials to enhance the bonding strength. Taking into account of the cost in industrial applications, $3 \%$ is a more economic choice.

\begin{tabular}{|c|c|c|c|c|c|c|}
\hline \multicolumn{7}{|c|}{$\begin{array}{c}\text { TABLE-1 } \\
\text { RESULTS OF ORTHOGONAL EXPERIMENT }\end{array}$} \\
\hline \multirow[t]{2}{*}{ No. } & $\mathrm{n}_{\mathrm{F}} / \mathrm{n}_{\mathrm{U}}$ & $\begin{array}{c}\mathrm{t}_{1} \\
(\mathrm{~min})\end{array}$ & $\mathrm{pH}$ & $\begin{array}{c}\mathrm{T} \\
\left({ }^{\circ} \mathrm{C}\right)\end{array}$ & $\begin{array}{c}\mathrm{t}_{2} \\
(\mathrm{~min})\end{array}$ & \multirow{2}{*}{$\begin{array}{c}\text { Free } \\
\text { formaldehyde } \\
\text { content }(\%)\end{array}$} \\
\hline & $\mathrm{A}$ & $\mathrm{B}$ & $\mathrm{C}$ & $\mathrm{D}$ & $\mathrm{E}$ & \\
\hline 1 & 1.0 & 20 & 3.5 & 65 & 20 & - \\
\hline 2 & 1.0 & 40 & 4.5 & 75 & 40 & 0.170 \\
\hline 3 & 1.0 & 60 & 5.5 & 85 & 60 & 0.240 \\
\hline 4 & 1.0 & 80 & 6.5 & 95 & 80 & 0.267 \\
\hline 5 & 1.1 & 80 & 4.5 & 85 & 20 & 0.229 \\
\hline 6 & 1.1 & 60 & 3.5 & 95 & 40 & - \\
\hline 7 & 1.1 & 40 & 6.5 & 65 & 60 & 0.419 \\
\hline 8 & 1.1 & 20 & 5.5 & 75 & 80 & 0.343 \\
\hline 9 & 1.2 & 40 & 5.5 & 95 & 20 & 0.406 \\
\hline 10 & 1.2 & 20 & 6.5 & 85 & 40 & 0.439 \\
\hline 11 & 1.2 & 80 & 3.5 & 75 & 60 & - \\
\hline 12 & 1.2 & 60 & 4.5 & 65 & 80 & 0.245 \\
\hline 13 & 1.3 & 60 & 6.5 & 75 & 20 & 0.520 \\
\hline 14 & 1.3 & 80 & 5.5 & 65 & 40 & 0.498 \\
\hline 15 & 1.3 & 20 & 4.5 & 95 & 60 & 0.341 \\
\hline 16 & 1.3 & 40 & 3.5 & 85 & 80 & - \\
\hline$\Sigma(1)$ & 0.677 & 1.123 & - & 1.162 & 1.155 & - \\
\hline$\Sigma(2)$ & 0.991 & 0.995 & 0.985 & 1.033 & 1.107 & - \\
\hline$\Sigma(3)$ & 1.090 & 1.005 & 1.487 & 0.908 & 1.000 & - \\
\hline$\Sigma(4)$ & 1.359 & 0.994 & 1.645 & 1.014 & 0.855 & Influence: \\
\hline $\mathrm{K} 1$ & 0.226 & 0.374 & - & 0.387 & 0.385 & $\mathrm{~A}>\mathrm{C}>\mathrm{E}>\mathrm{D}>\mathrm{B}$ \\
\hline $\mathrm{K} 2$ & 0.330 & 0.332 & 0.246 & 0.344 & 0.369 & - \\
\hline K3 & 0.363 & 0.335 & 0.372 & 0.303 & 0.333 & - \\
\hline K4 & 0.453 & 0.331 & 0.411 & 0.338 & 0.285 & - \\
\hline $\mathrm{R}$ & 0.227 & 0.043 & 0.165 & 0.084 & 0.100 & - \\
\hline
\end{tabular}

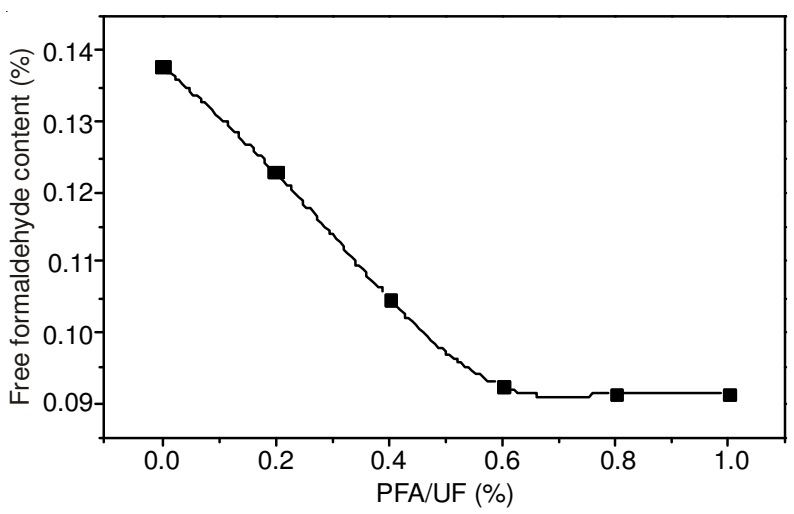

Fig. 1. Free formaldehyde content of UF resins with the PFA/UF

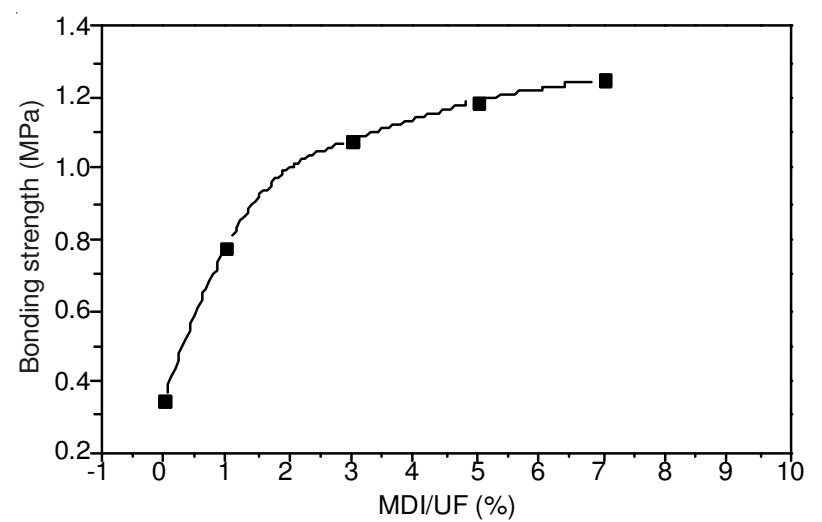

Fig. 2. Bonding strength of UF resins with second part of MDI/UF

FT-IR analysis: Samples of FA, PFA, UF and PMUF were analyzed by FTIR spectrometer of 6700 NICOLET with ATR 
method. Fig. 3 showed the strong absorption peaks between $3400-3200 \mathrm{~cm}^{-1}$ is $-\mathrm{OH}$ vibration absorption; $1600 \mathrm{~cm}^{-1}$ is aromatic ring around skeleton $\mathrm{C}=\mathrm{C}$ vibration absorption; 1040 $\mathrm{cm}^{-1}$ is C-O telescopic vibration absorption peaks; $755 \mathrm{~cm}^{-1}$ is a new absorption of PFA at the result of the reaction between $\mathrm{C}-\mathrm{H}$ of phenol and $\alpha-\mathrm{OH}$ of fulvic acid. So PFA contains more active site to react with formaldehyde.

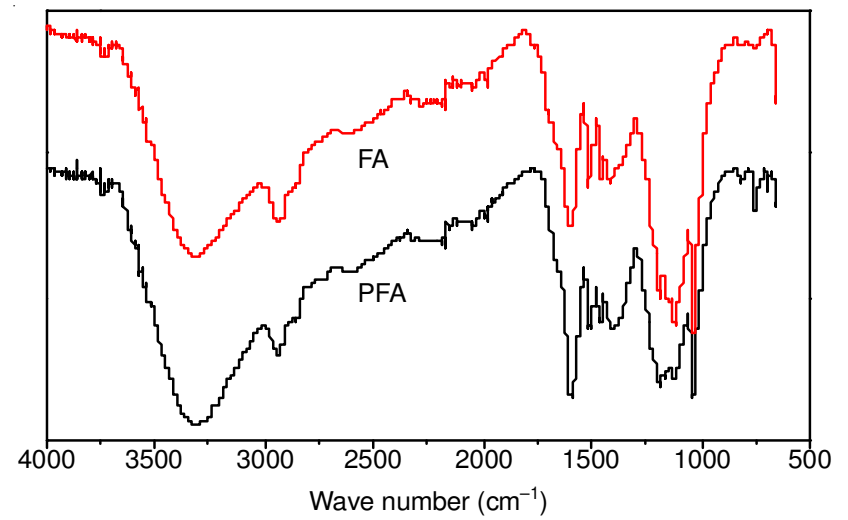

Fig. 3. FT-IR spectra of FA and PFA

Fig. 4 showed the strong absorption peaks between 3500$3200 \mathrm{~cm}^{-1}$ caused by $-\mathrm{OH}$ and $-\mathrm{NH}_{2}$ associating action; 1597 $\mathrm{cm}^{-1}$ is $\mathrm{C}=\mathrm{C}$ of aromatic ring and $\mathrm{C}=\mathrm{O}$ associating absorption; $1132 \mathrm{~cm}^{-1}$ is C-O-C telescopic vibration absorption; $776 \mathrm{~cm}^{-1}$ is Ar-H vibration absorption peaks, a new absorption of PMUF which shows that some substitution reactions were reacted on the benzene ring. So the PFA and resins were combined with chemical bonds.

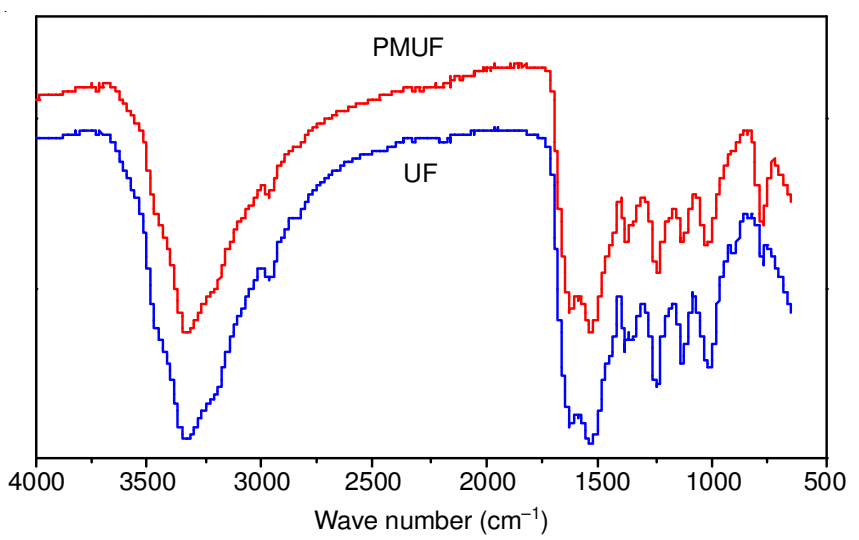

Fig. 4. FT-IR spectra of UF and PMUF

TG and DSC analysis: Samples of UF and PMUF were analyzed by simultaneous thermal analyzer of STA409PC Netzsch with heating rate $10^{\circ} \mathrm{C} / \mathrm{min}$ from $40-800{ }^{\circ} \mathrm{C}$. Fig. 5 shows rapid decline before $100^{\circ} \mathrm{C}$, it is mainly because water molecules were evaporated, which explains the strong endothermic peak of DSC curves before $100{ }^{\circ} \mathrm{C}$ in Fig. 6. The slow decline of TG curves between 100 and $200{ }^{\circ} \mathrm{C}$ are caused by the evaporation of by-product of polycondensation reaction and emission of formaldehyde from the break of unstable ether bond. The rapid decline of TG curves between 200 and 350 ${ }^{\circ} \mathrm{C}$ are caused by degradation of resin structure, which explains the endothermic and exothermic peaks of the DSC curves.

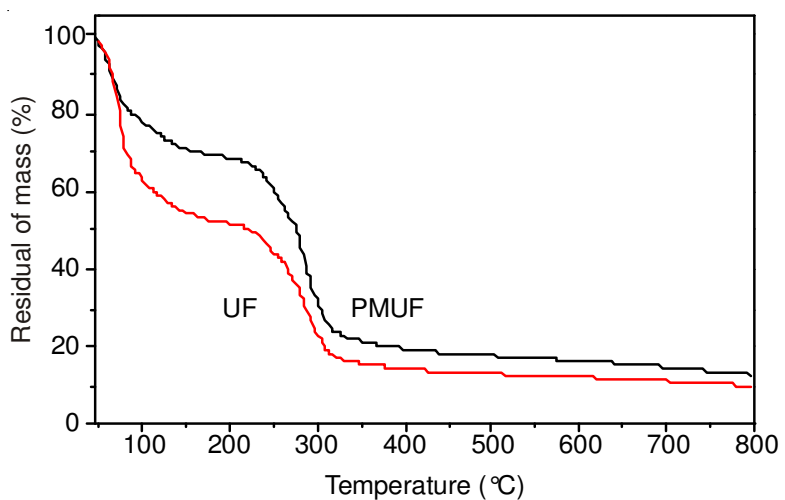

Fig. 5. TG curves of UF and PMUF

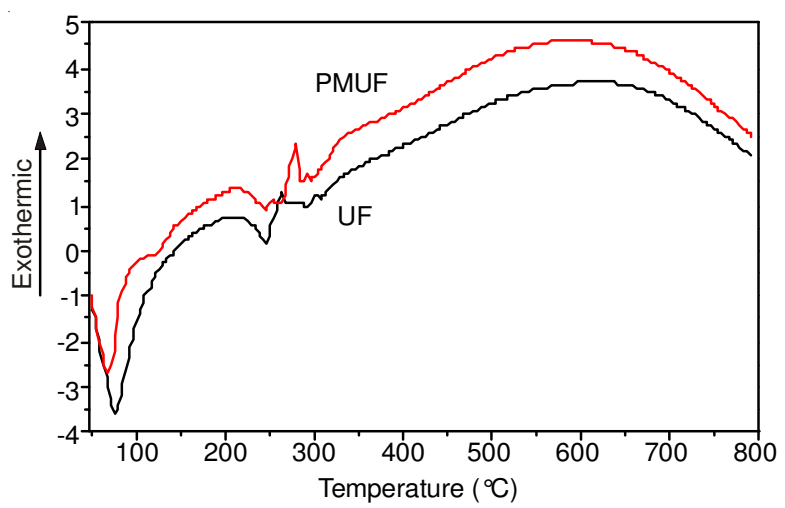

Fig. 6. DSC curves of UF and PMUF

Residual of mass are little changed after $350^{\circ} \mathrm{C}$, which indicates that the resins were carbonized and there are carbonization exothermic peak of DSC curves. According to the TG curves, PMUF obtains higher residual of mass at the same temperature. So the thermal stability of resins was improved after modification.

\section{Conclusion}

Phenolized fulvic acid contains more active site to react with formaldehyde than FA and it will be able to reduce the content of free formaldehyde of UF resins. The optimal conditions are as follows: $\mathrm{n}_{\mathrm{F}} / \mathrm{n}_{\mathrm{U}}=1.0, \mathrm{t}_{1}=80 \mathrm{~min}, \mathrm{pH}=4.5, \mathrm{~T}=$ $85^{\circ} \mathrm{C}, \mathrm{t}_{2}=80 \mathrm{~min}, 0.6 \%$ PFA, $3.5 \% \mathrm{MDI}$. The content of the free formaldehyde is dropped to $0.0924 \%$, which has a huge advantage of $0.2 \%$ for sale in the market. The bonding strength can achieve at $1.08 \mathrm{MPa}$ and the thermal stability is also improved. This study indicates that the content of free formaldehyde and the bonding strength of UF resins could get better at the same time with a special condition.

\section{REFERENCES}

1. C.W. Bayer and M.S. Black, Exp. Pathol., 37, 147 (1989).

2. M. Dunky, Int. J. Adhes. Adhes., 18, 95 (1998).

3. I. Andersen, G.R. Lundqvist and L. Mølhave, Atmos. Environ., 9, 1121 (1975).

4. M. David, Regul. Toxicol. Pharmacol., 4, 355 (1983).

5. A.P. Jones, Atmos. Environ., 33, 4535 (1999).

6. Z. Que, T. Furuno, S. Katoh and Y. Nishino, Build. Environ., 42, 1257 (2007).

7. Z. Que, T. Furuno, S. Katoh and Y. Nishino, Build. Environ., 42, 1242 (2007).

8. A. Pizzi, L. Lipschitz and J. Valenzuela, Holzforschung, 48, 254 (1994).

9. E.M. George, Forest Prod. J., 5, 35 (1984). 